\title{
Performance Evaluation of Distance-Hop Proportionality on Geometric Graph Models of Dense Sensor Networks
}

\author{
Swaprava Nath and Anurag Kumar \\ Dept. of Electrical Communication Engineering \\ Indian Institute of Science, Bangalore 560012, INDIA \\ swaprava@gmail.com, anurag@ece.iisc.ernet.in
}

\begin{abstract}
Wireless sensor networks can often be viewed in terms of a uniform deployment of a large number of nodes on a region in Euclidean space, e.g., the unit square. After deployment, the nodes self-organise into a mesh topology. In a dense, homogeneous deployment, a frequently used approximation is to take the hop distance between nodes to be proportional to the Euclidean distance between them. In this paper, we analyse the performance of this approximation. We show that nodes with a certain hop distance from a fixed anchor node lie within a certain annulus with probability approaching unity as the number of nodes $n \rightarrow \infty$.

We take a uniform, i.i.d. deployment of $n$ nodes on a unit square, and consider the geometric graph on these nodes with radius $r(n)=c \sqrt{\frac{\ln n}{n}}$. We show that, for a given hop distance $h$ of a node from a fixed anchor on the unit square, the Euclidean distance lies within $[(1-\epsilon)(h-1) r(n), h r(n)]$, for $\epsilon>0$, with probability approaching unity as $n \rightarrow \infty$. This result shows that it is more likely to expect a node, with hop distance $h$ from the anchor, to lie within this annulus centred at the anchor location, and of width roughly $r(n)$, which decreases as $n$ increases. We show that if the radius $r$ of the geometric graph is fixed, the convergence of the probability is exponentially fast. Similar results hold for a randomised lattice deployment. We provide simulation results that illustrate the theory, and serve to show how large $n$ needs to be for the asymptotics to be useful.
\end{abstract}

\section{INTRODUCTION}

We consider a dense wireless sensor network comprising a large number of nodes, $n$, distributed over a region in Euclidean space, e.g., the unit square. If the communication range of every node is $r$, the communication topology becomes a geometric graph, i.e., each node is connected to every other node that is at a distance $\leq r$. If the node deployment is random in some sense, e.g., uniform i.i.d. deployment, then the network topology becomes a random geomet-

Permission to make digital or hard copies of all or part of this work for personal or classroom use is granted without fee provided that copies are not made or distributed for profit or commercial advantage and that copies bear this notice and the full citation on the first page. To copy otherwise, to republish, to post on servers or to redistribute to lists, requires prior specific permission and/or a fee.

Valuetools 2008, October 21-23, 2008, Athens, GREECE

Copyright 2008 ICST ISBN \# 978-963-9799-31-8 . ric graph (RGG) (see, e.g., [7]). Given a dense deployment of nodes, and a topology over them, a frequently used approximation is to take the minimum number of hops between nodes (i.e., the hop distance) as a measure of the Euclidean distance between them. Niculescu and Nath [6], Nagpal et al. [4] and Yang et al. [9] have used this approximation to develop techniques for GPS-free localisation in dense wireless sensor networks. Yang et al., in particular, make a key assumption that the ratio of the Euclidean distance between a node and two anchor nodes is well approximated by the ratio of the corresponding hop distances.

The relation between the number of hops and the Euclidean distance traversed has been studied analytically in previous literature. Vural and Ekici [8] have considered an RGG with radius $r$, in one dimension. They have studied the distribution of the maximum possible Euclidean distance travelled along a line, by a path of a given number of hops, and have obtained approximations to the mean and variance of these distributions. A similar analysis has been performed by Dulman et al. [1], where two dimensional node deployments have been considered in some detail. To the best of our knowledge, the approximation of proportionality between Euclidean and Hop-distance in a dense network still lacks theoretical formalisation. This has been pointed out in a recent paper by $\mathrm{Li}$ and $\mathrm{Liu}$ [3], where the authors consider this as a heuristic even for an isotropic network. We attempt to formalise this notion of proportionality.

In Section 3, we find bounds on Euclidean distance for a given hop distance on arbitrary geometric graphs in 2 dimensions, and show that for 2-D, the hop distance (HD) is not a good measure of Euclidean distance (ED). However, when the node deployment is random, thus yielding an RGG, HD does become proportional to ED in an approximate sense. This has been explored in the sections to follow. For the RGG, nodes are distributed in a uniform i.i.d. fashion over a unit area $\mathcal{A} \subset \mathbb{R}^{2}$, i.e., the location of each node is uniformly distributed over $\mathcal{A}$, independent of the locations of the other nodes. On such a deployment of nodes, we consider the RGG with radius $r(n)=c \sqrt{\frac{\ln n}{n}}, c>\frac{1}{\sqrt{\pi}}$, which ensures connectedness of the RGG with probability approaching 1 , as $n \rightarrow \infty$ (by Gupta and Kumar [2]). We find in Section 4 that given a HD $h$ from an anchor node (location fixed) on this RGG, the ED $d$ between the anchor and nodes that are $h$ hops from the anchor lies within an interval $[(1-\epsilon)(h-1) r(n), h r(n)]$ w.h.p. ${ }^{1}$, for $\epsilon>0$, with the

\footnotetext{
${ }^{1}$ w.h.p. (with high probability) means that the probability of the said event $\rightarrow 1$ as $n \rightarrow \infty$
} 


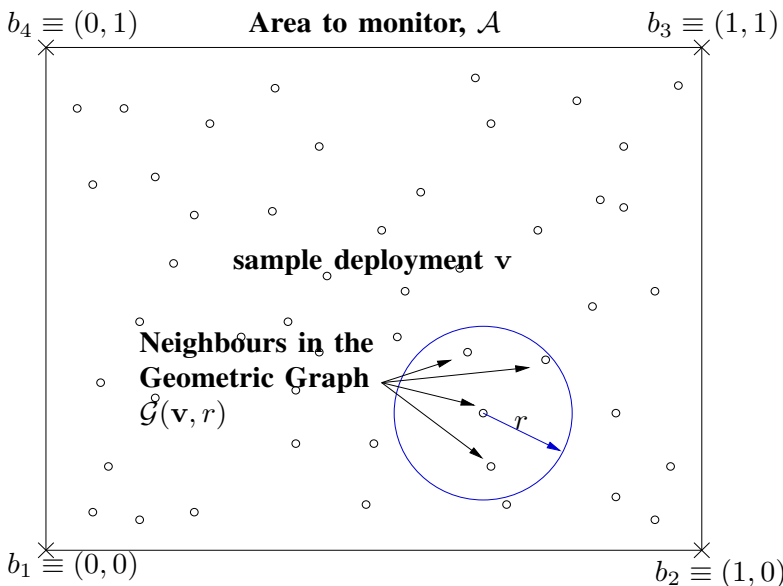

Node locations can be arbitrary or random

Figure 1: An example deployment with 4 anchors.

convergence rate dictated by the $\epsilon$ chosen. This result gives a theoretical justification for the ED-HD proportionality assumptions of [6] and [9] for $h>>1$ and large $n$. In Section 5, we show that the rate of convergence can be improved if the radius $r$ does not vary with $n$. Of course, we need to choose $n$ large enough so that the radius for connectivity according to [2] is smaller than $r$. We extend both the results for the case of randomised lattice deployment ${ }^{2}$ in Section 6 .

Our results are via bounds and provide a sufficient condition for the rate of convergence. However in Section 7, we have considered Poisson deployment in 1-dimension for which these conditions are necessary and sufficient. Finally in Section 8, we provide simulation results to illustrate the theorems.

\section{THE GEOMETRIC GRAPH SETTING \& NOTATION}

In this section we describe the basic setting for our results, and also develop the main notation.

\section{Setting:}

- $n$ nodes are deployed on a unit 2-dimensional area $\mathcal{A}$. The node locations are denoted by the vector $\mathbf{v}=$ $\left[v_{1}, v_{2}, \cdots, v_{n}\right] \in \mathcal{A}^{n}$, where $v_{i}$ is the location of the $i^{\text {th }}$ node.

- We form the geometric graph $\mathcal{G}(\mathbf{v}, r)$ by connecting nodes that are within the distance $r$ of each other. Then $r$ is called the radius of the geometric graph.

We define anchors as nodes whose locations are known $a$ priori, e.g., in Figure 1, we have shown 4 anchors $b_{1}, b_{2}, b_{3}$ and $b_{4}$, with their positions fixed at the 4 corners of the unit square $\mathcal{A}$.

${ }^{2} \mathrm{~A}$ randomised lattice deployment is obtained as follows. The area $\mathcal{A}$ is partitioned into $n$ equal area "cells", e.g., squares, and one node is placed at a uniformly distributed random location in each cell.

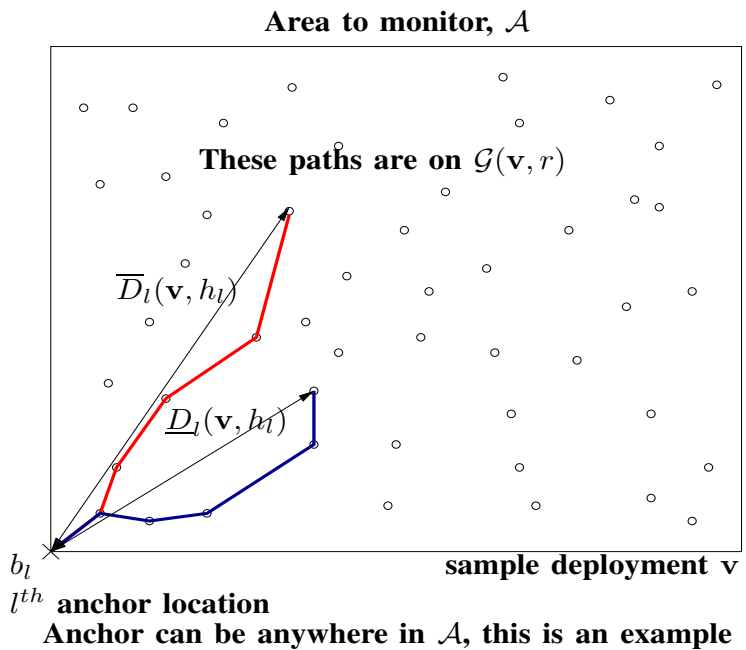

Figure 2: Graphical illustration of $\bar{D}_{l}\left(\mathbf{v}, h_{l}\right)$ and $\underline{D}_{l}\left(\mathbf{v}, h_{l}\right)\left(h_{l}=5\right.$ in the illustration $)$.

\section{Notation:}

- $\mathcal{N}=[n]=\{1,2, \cdots, n\}$, the index set of the nodes, i.e., node $i \in \mathcal{N}$ has a location $v_{i}$ on $\mathcal{A}$.

- $b_{l}=$ Location of the $l^{t h}$ anchor node, $l=1, \cdots, L$, e.g., in Figure 1, $L=4$.

- $H_{l, i}(\mathbf{v})=$ Minimum number of hops of node $i$ from anchor $b_{l}$ on the graph $\mathcal{G}(\mathbf{v}, r)$ for the deployment $\mathbf{v}$.

- $D_{l, i}(\mathbf{v})=$ Euclidean distance of node $i$ from anchor $b_{l}$ for the deployment $\mathbf{v}$.

$$
\begin{aligned}
& \bar{D}_{l}\left(\mathbf{v}, h_{l}\right)=\max _{\left\{i \in \mathcal{N}: H_{l, i}(\mathbf{v})=h_{l}\right\}} D_{l, i}(\mathbf{v}) \\
& \underline{D}_{l}\left(\mathbf{v}, h_{l}\right)=\min _{\left\{i \in \mathcal{N}: H_{l, i}(\mathbf{v})=h_{l}\right\}} D_{l, i}(\mathbf{v})
\end{aligned}
$$

A graphical illustration of the above two quantities is given in Figure 2.

- For all $j \in \mathcal{N}, \mathcal{N}_{j}=\left\{k \in \mathcal{N}:\left\|v_{j}-v_{k}\right\| \leq r, k \neq j\right\}$. This is the neighbour set of node $j$ in $\mathcal{G}(\mathbf{v}, r)$.

With this setting, given the hop distance $h_{l}$ on $\mathcal{G}(\mathbf{v}, r)$ between a node and an anchor, we wish to obtain constraints on the Euclidean distance of the node from anchor $b_{l}$.

\section{HD-ED RELATIONSHIP IN ARBITRARY GEOMETRIC GRAPHS}

In this section, we evaluate the performance of distancehop proportionality in an arbitrary geometric graph with radius $r$, where by "arbitrary" we mean that the node locations are arbitrary. We show that the proportionality approximation can be arbitrarily coarse.

We define a sequence of nodes $\left\{a_{1}, a_{2}, \cdots, a_{K}\right\}$, where all $a_{i} \in \mathcal{N}, i=1, \cdots, K$, as sequential neighbours iff

$$
\mathcal{N}_{a_{i}}= \begin{cases}\left\{a_{i-1}, a_{i+1}\right\} & \text { for } i=2, \cdots, K-1 \\ \left\{a_{i+1}\right\} & \text { for } i=1 \\ \left\{a_{i-1}\right\} & \text { for } i=K\end{cases}
$$




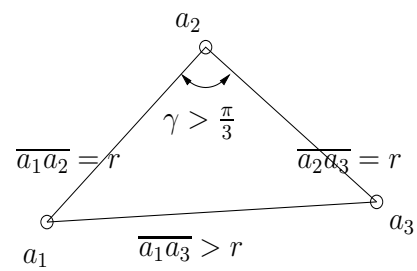

Figure 3: Condition for sequential neighbours in arbitrary geometric graph.

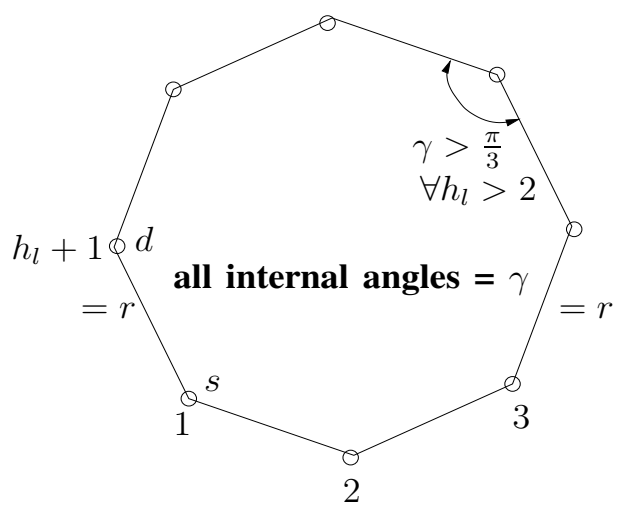

A regular $h_{l}+1$ sided polygon

Figure 4: Construction to find the lower bound on Euclidean distance.

We can observe if $\left\{a_{1}, a_{2}, a_{3}\right\}$ have the following properties, $\overline{a_{1} a_{2}}=r, \overline{a_{2} a_{3}}=r$ and $\overline{a_{1} a_{3}}>r,{ }^{3}$ they will be sequential neighbours on $\mathcal{G}(\mathbf{v}, r)$ (See Figure 3 ). Then, by using cosine law in $\triangle a_{1} a_{2} a_{3}$, we get, $\overline{a_{1} a_{3}}=\sqrt{r^{2}+r^{2}-2 r \cdot r \cos \gamma}=$ $r \sqrt{2(1-\cos \gamma)}>r$, for $\gamma>\frac{\pi}{3}$. Now, to find a bound on ED for HD $h_{l}$, we construct a regular polygon with $h_{l}+1$ sides, all with length $r$, as shown in Figure 4 . We know that the total interior angle is $\left(h_{l}+1-2\right) \pi=\left(h_{l}-1\right) \pi$. Hence each angle is $\frac{\left(h_{l}-1\right) \pi}{h_{l}+1}$. We see that, $\frac{\left(h_{l}-1\right) \pi}{h_{l}+1}>\frac{\pi}{3}$, iff $h_{l}>2$. So, for all hop distances $h_{l} \geq 3$, each of the internal angles will be $>\frac{\pi}{3}$. Now we pick two adjacent nodes $s$ and $d$ as shown in Figure 4. We want the hop distance between them to be $h_{l}$, we delete the edge $s d$ and increase all the other angles by a very small amount $\delta$, hence we get the node sequence as shown in Figure 5. This sequence of nodes will be sequential neighbours iff the ED between $s$ and $d$, i.e. $r_{1}$, becomes $>r$. Now the following lemma says that for a certain choice of $\delta, r_{1}>r$ with the nodes on the path from $s$ to $d$ being sequential neighbours.

Lemma 1. For $h_{l}>2$ and $0<\delta<\frac{4 \pi}{h_{l}+1}, r_{1}>r$

Proof: In Appendix.

Hence $s$ and $d$ cease to be neighbours and the nodes in the path from $s$ to $d$ still follow the properties of being sequential neighbours. Assuming other nodes to be more than $r$ from all the nodes of this set of $h_{l}+1$ nodes, the hop distance between $s$ and $d$ becomes $h_{l}$, however the Euclidean distance

$\overline{{ }^{3} \text { Define, } \overline{a_{j} a_{k}}=\left\|v_{a_{j}}-v_{a_{k}}\right\|}$

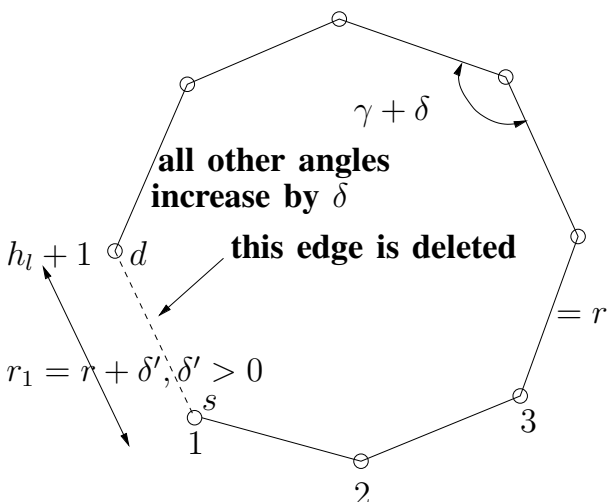

hop distance between $s$ and $d=h_{l}$

Figure 5: Achievability of the lower bound.

between them is just more than $r$, for $h_{l} \geq 3$. For $h_{l}=2$, a construction similar to Figure 3 can be done to show that the distance between $s=a_{1}$ and $d=a_{3}$ is just a little more than $r$. Hence for any arbitrary geometric graph in 2-dimensions, given the hop distance between a node and an anchor being $h_{l} \geq 2$, the Euclidean distance can be arbitrarily close but more than $r$, which is a trivial lower bound. The upper bound on ED remains $h_{l} r$ as usual, which can be achieved by placing the nodes on a straight line $r$ distance from each other to form a set of $h_{l}+1$ sequential neighbours. Hence, we have proved the following lemma.

Lemma 2. For arbitrary $\mathbf{v}$ and $h_{l} \geq 2, r<\underline{D}_{l}\left(\mathbf{v}, h_{l}\right) \leq$ $\bar{D}_{l}\left(\mathbf{v}, h_{l}\right) \leq h_{l} r$ and both bounds are sharp.

Hence the hop distance in an arbitrary geometric graph on a plane does not provide useful information about the Euclidean distance between the nodes.

However, the situation changes when the distribution of nodes has positive density over all points on $\mathcal{A}$, e.g., the node distribution is uniform i.i.d. or randomised lattice. As we will find out in the following sections, in a random geometric graph with a sufficient number of nodes, the hop-distance serves as a good measure of the Euclidean distance.

\section{HD-ED RELATIONSHIP IN RANDOM GE- OMETRIC GRAPHS}

In this section we will provide theoretical results concerning distance-hop proportionality in an RGG. Thus, we now specialise to the following setting.

\section{Setting:}

- $n$ nodes are deployed on a unit area $\mathcal{A}$ in the uniform i.i.d. fashion. The difference with this setup from the previous section is that the node locations are random, and are denoted by the random vector $\mathbf{V} \in \mathcal{A}^{n}$, with a particular realisation being denoted by $\mathbf{v}$. We denote by $\mathbb{P}^{n}($.$) the probability measure on \mathcal{A}^{n}$ so obtained.

- We form the RGG $\mathcal{G}(\mathbf{v}, r(n))$ by connecting the nodes that are within the radius $r(n)$ of each other, where $r(n)$, the radius of the geometric graph is chosen so 
that the network remains asymptotically connected. We take $r(n)=c \sqrt{\frac{\ln n}{n}}, c>\frac{1}{\sqrt{\pi}}$, a constant; this ensures asymptotic connectivity (see [2]).

In Section 4.1, we analyse the distribution of distance from one anchor node and in Section 4.2, we generalise it for $L$ anchors.

The choice of the radius, $r(n)=c \sqrt{\frac{\ln n}{n}}, c>\frac{1}{\sqrt{\pi}}$, does not only guarantee asymptotic connectivity among the nodes, but also ensures connectivity of the nodes with all the anchors. The following lemma states that there will be at least a node within a distance $r(n)$ of each anchor $b_{l}, l=1, \cdots, L$ w.h.p. and so the nodes are connected to all the anchors in a dense network. Define, $B_{l}=\{\mathbf{v}: \exists$ at least one node within a radius of $r(n)$ from $\left.b_{l}\right\}, l=1, \cdots, L$.

Lemma 3. $\lim _{n \rightarrow \infty} \mathbb{P}^{n}\left(\cap_{l=1}^{L} B_{l}\right)=1$

Proof: $\mathbb{P}^{n}\left(\cap_{l=1}^{L} B_{l}\right)=1-\mathbb{P}^{n}\left(\cup_{l=1}^{L} B_{l}^{c}\right) \geq 1-\sum_{l=1}^{L} \mathbb{P}^{n}\left\{B_{l}^{c}\right\}=$

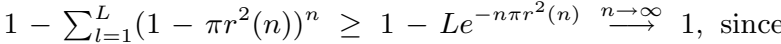
$r(n)=c \sqrt{\frac{\ln n}{n}}$ and $1-x \leq e^{-x}$.

\subsection{Distance distribution from a fixed anchor $b_{l}$ : Uniform i.i.d. deployment}

We make the construction as shown in Figure 6. From $b_{l}$ (without loss of generality, we can choose $l=1$ ), we draw a circle of radius $h_{l} r(n)$ centred at $b_{l}$, this is the maximum distance reachable in $h_{l}$ hops, by triangle inequality, since each hop can be of maximum length $r(n)$. All the nodes $\{i \in$ $\left.\mathcal{N}: H_{l, i}(\mathbf{v})=h_{l}\right\}$ lie within this disk. So, $\bar{D}_{l}\left(\mathbf{v}, h_{l}\right) \leq h_{l} r(n)$ for all $\mathbf{v}$. To obtain a lower bound on $\underline{D}_{l}\left(\mathbf{v}, h_{l}\right)$, we construct blades as shown in Figure 6 . We start with one blade. It will cover some portion of the circumference of the circle of radius $h_{l} r(n)$; see Figure 6 . Construct the next blade so that it covers the adjacent portion of the circumference that has not been covered by the previous blade. We go on constructing these blades until the entire portion of the circle lying inside the unit square $\mathcal{A}$ is covered (see Figure 6). Let us define,

- $J(n)$ : Number of blades required to cover the part of the circle within $\mathcal{A}$

- $\mathcal{B}_{j}^{l}: j^{\text {th }}$ blade drawn from the point $b_{l}$ as shown in Figure $6,1 \leq j \leq J(n)$.

On each of these blades, we construct $h_{l}$ strips, shown shaded in Figure 7, $u(n)$ being the width of the blade and $t(n)$ the width of the strip. We define the following event.

- $A_{i, j}^{l}=\left\{\mathbf{v}: \exists\right.$ at least one node in the $i^{\text {th }}$ strip of $\left.\mathcal{B}_{j}^{l}\right\}$

If a $\mathbf{v} \in A_{i, j}^{l}, \forall 1 \leq i \leq h_{l}-1,1 \leq j \leq J(n)$, i.e., there exists at least one node in each of the $h_{l}-1$ strips (see Figure 7) for all the blades $\mathcal{B}_{j}^{l}$, then for that $\mathbf{v}$, all nodes at a distance $<(p-q)\left(h_{l}-1\right) r(n)$ from $b_{l}$ are reachable in at most $h_{l}-1$ hops, hence will have a hop distance $\leq h_{l}-1<$ $h_{l}$. So, we have $\underline{D}_{l}\left(\mathbf{v}, h_{l}\right) \geq(p-q)\left(h_{l}-1\right) r(n)$, for such a deployment v; see Figure 7. Hence,

$$
\begin{aligned}
& \left\{\cap_{j=1}^{J(n)} \cap_{i=1}^{h_{l}-1} A_{i, j}^{l}\right\} \\
& \quad \subseteq \quad\left\{\mathbf{v}:(p-q)\left(h_{l}-1\right) r(n) \leq \underline{D}_{l}\left(\mathbf{v}, h_{l}\right)\right. \\
& \left.\quad \leq \bar{D}_{l}\left(\mathbf{v}, h_{l}\right) \leq h_{l} r(n)\right\}
\end{aligned}
$$

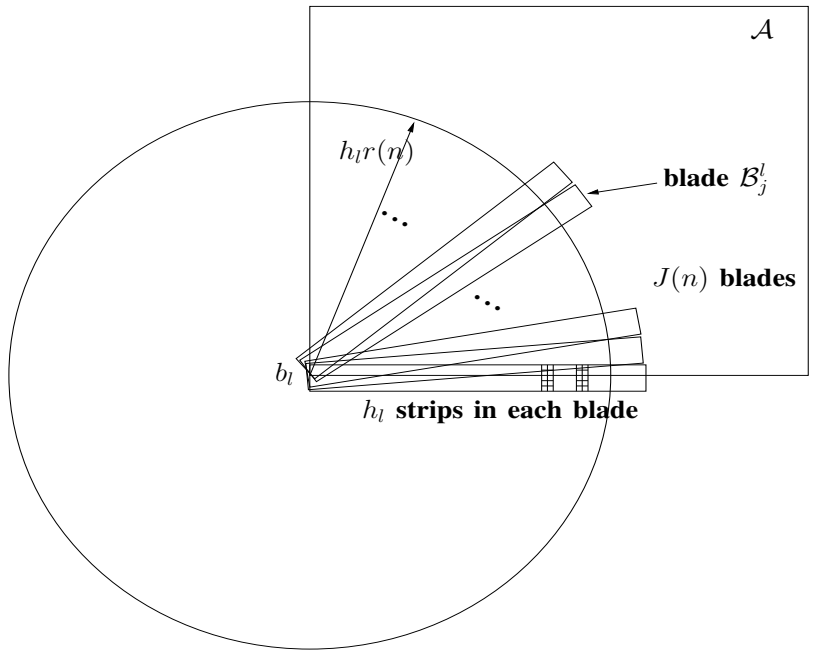

Figure 6: Construction using the blades cutting the circumference of the circle of radius $h_{l} r(n)$.

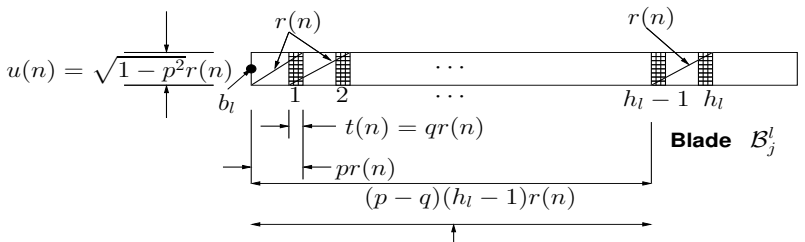

all nodes that fall here will have hop distance $\leq h_{l}-1$ from $b_{l}$

Figure 7: The construction with $h_{l}$ hops.

Since $1>p>q>0$, we can choose $p-q$ to be equal to $1-\epsilon$, for the given $\epsilon>0$. So the lower bound in Equation 1 becomes, $(p-q)\left(h_{l}-1\right) r(n)=(1-\epsilon)\left(h_{l}-1\right) r(n)$.

To find the value of $J(n)$, we need to define the following.

- $a(n)$ is the length of the arc of radius $h_{l} r(n)$ that lies within a blade, drawn taking $b_{l}$ as centre, as shown in Figure 8.

- $\alpha(n)$ : angle subtended by $a(n)$ at $b_{l}$, see Figure 8 .

Now from Figure 6, we have, $J(n)=\left[\frac{\pi}{2 \alpha(n)}\right]$. We also have from Figure $8, h_{l} r(n) \alpha(n)=a(n) \geq u(n)=\sqrt{1-p^{2}} r(n)$. Hence, $\alpha(n) \geq \frac{\sqrt{1-p^{2}}}{h_{l}}$. So, $J(n) \leq\left\lceil\frac{\pi h_{l}}{2 \sqrt{1-p^{2}}}\right\rceil$. 


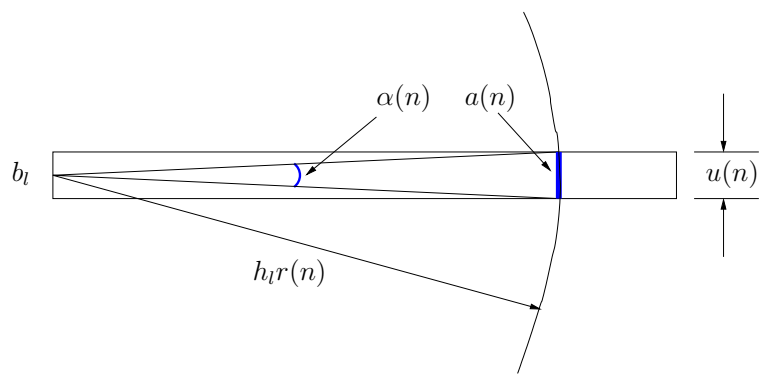

Figure 8: Construction to find $J(n)$.

Now we compute,

$$
\begin{aligned}
\mathbb{P}^{n} & \left(\cap_{j=1}^{J(n)} \cap_{i=1}^{h_{l}-1} A_{i, j}^{l}\right) \\
& =1-\mathbb{P}^{n}\left(\cup_{j=1}^{J(n)} \cup_{i=1}^{h_{l}-1} A_{i, j}^{l}{ }^{c}\right) \\
& \geq 1-\sum_{j=1}^{J(n)} \sum_{i=1}^{h_{l}-1} \mathbb{P}^{n}\left(A_{i, j}^{l}{ }^{c}\right) \\
& \geq 1-\left\lceil\frac{\pi h_{l}}{2 \sqrt{1-p^{2}}}\right\rceil\left(h_{l}-1\right)(1-u(n) t(n))^{n} \\
& \geq 1-\left\lceil\frac{\pi h_{l}}{2 \sqrt{1-p^{2}}}\right\rceil\left(h_{l}-1\right) e^{-n u(n) t(n)} \\
& =1-\left\lceil\frac{\pi h_{l}}{2 \sqrt{1-p^{2}}}\right\rceil\left(h_{l}-1\right) e^{-n q \sqrt{1-p^{2}} r^{2}(n)} \\
& \stackrel{n \rightarrow \infty}{\longrightarrow} 1
\end{aligned}
$$

The first inequality comes from the union bound, the second inequality, from the upper bound on $J(n)$. The third inequality uses the result $1-x \leq e^{-x}$. We see that if the node distribution was non-homogeneous with positive density over all points in $\mathcal{A}$, the term $(1-u(n) t(n))^{n}$ could have been replaced by $\left(1-f_{\min } u(n) t(n)\right)^{n}$, where $f_{\min }$ is the minimum density over $\mathcal{A}$ and as $f_{\min }>0$, the same convergence result would be true even for non-homogeneous node placement.

Let us define, $E_{h_{l}}(n)=\left\{\mathbf{v}:(1-\epsilon)\left(h_{l}-1\right) r(n) \leq \underline{D}_{l}\left(\mathbf{v}, h_{l}\right)\right.$ $\left.\leq \bar{D}_{l}\left(\mathbf{v}, h_{l}\right) \leq h_{l} r(n)\right\}$. So, we have, for the given $\epsilon>0$, and using Equations 1 and 2,

$$
\begin{aligned}
1 & \geq \mathbb{P}^{n}\left(E_{h_{l}}(n)\right) \\
& \geq \mathbb{P}^{n}\left(\cap_{j=1}^{J(n)} \cap_{i=1}^{h_{l}-1} A_{i, j}^{l}\right) \\
& \geq 1-\left(h_{l}-1\right)\left[\frac{\pi h_{l}}{2 \sqrt{1-p^{2}}}\right] e^{-n q \sqrt{1-p^{2}} c^{2} \frac{\ln n}{n}}
\end{aligned}
$$

which implies,

$0 \leq 1-\mathbb{P}^{n}\left(E_{h_{l}}(n)\right) \leq\left(h_{l}-1\right)\left\lceil\frac{\pi h_{l}}{2 \sqrt{1-p^{2}}}\right\rceil e^{-q \sqrt{1-p^{2}} c^{2} \ln n}$

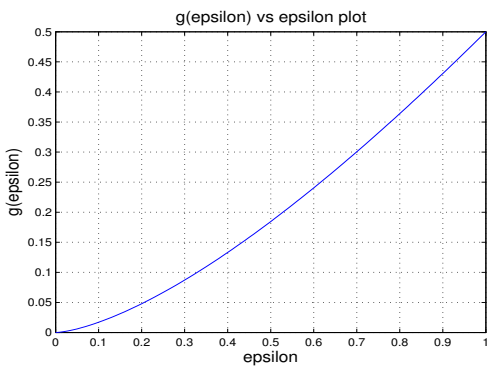

Figure 9: $g(\epsilon)$ vs $\epsilon$ plot.

And as $n \rightarrow \infty$,

$$
\begin{aligned}
1 & -\mathbb{P}^{n}\left(E_{h_{l}}(n)\right) \\
& =\mathcal{O}\left(e^{-q \sqrt{1-p^{2}} c^{2} \ln n}\right) \\
& =\mathcal{O}\left(\frac{1}{n^{q \sqrt{1-p^{2}} c^{2}}}\right)
\end{aligned}
$$

This result is true for any $p$ and $q$. But we can choose these constants so that the convergence $\rightarrow 0$ of the bound in Equation 5 is the most rapid, i.e., $p$ and $q$ are chosen so as to maximise $q \sqrt{1-p^{2}}$, thus making the upper bound to reduce at the fastest rate. For the given $\epsilon>0, p-q=$ $1-\epsilon \Rightarrow q=p-(1-\epsilon)$. We can show that, $p=\arg \max _{p}(p-$ $(1-\epsilon)) \sqrt{1-p^{2}}=\frac{1-\epsilon+\sqrt{(1-\epsilon)^{2}+8}}{4}, q=\frac{-3(1-\epsilon)+\sqrt{(1-\epsilon)^{2}+8}}{4}$. Then writing, $g(\epsilon)=q(\epsilon) \sqrt{1-p^{2}(\epsilon)}$, we obtain the following theorem,

TheOrem 1. For a given $1>\epsilon>0$, and $r(n)=c \sqrt{\frac{\ln n}{n}}$, $c>\frac{1}{\sqrt{\pi}}, \mathbb{P}^{n}\left(E_{h_{l}}(n)\right)=1-\mathcal{O}\left(\frac{1}{n^{g(\epsilon) c^{2}}}\right)$,

where $g(\epsilon)=q(\epsilon) \sqrt{1-p^{2}(\epsilon)}$,

$p(\epsilon)=\frac{1-\epsilon+\sqrt{(1-\epsilon)^{2}+8}}{4}, q(\epsilon)=\frac{-3(1-\epsilon)+\sqrt{(1-\epsilon)^{2}+8}}{4}$.

Remark: A plot of $g(\epsilon)$ vs $\epsilon$ is given in Figure 9. We see that $g(\epsilon) \downarrow 0$ as $\epsilon \downarrow 0$. Hence Theorem 1 says that $\lim _{n \rightarrow \infty} \mathbb{P}^{n}\left(E_{h_{l}}(n)\right)=1$, for any $1>\epsilon>0$, so we can expect a node having a HD of $h_{l}$ from anchor $b_{l}$ to be within a distance $\left[(1-\epsilon)\left(h_{l}-1\right) r(n), h_{l} r(n)\right]$ from $b_{l}$ in a dense network. We notice that the width of this band of uncertainty is roughly $r(n)$, which is the unit of distance measurement on $\mathcal{G}(\mathbf{v}, r(n))$. The theorem also says that the rate of convergence is governed by the $\epsilon$ chosen, i.e., the smaller the $\epsilon$, the slower the rate of convergence.

\subsection{Distance distribution from fixed anchors $b_{l}, l=1, \cdots, L$ : Uniform i.i.d. deployment}

For $L$ anchors, the question arises whether the hop distances from the $L$ anchors are feasible or not, e.g., if we denote a disk with centre $a$ and radius $r$, by $C(a, r)=\{z \in$ $\mathcal{A}:\|z-a\| \leq r\}$, then a necessary condition for a feasible $\mathbf{h}$ vector $\left(\mathbf{h}=\left[h_{1}, \cdots, h_{l}, \cdots, h_{L}\right] \in \mathbb{N}^{L}\right.$ is the hop distance vector) is that $\cap_{l=1}^{L} C\left(b_{l}, h_{l} r(n)\right) \neq \phi$ (there will be other feasibility conditions also). We denote the set of all feasible $\mathbf{h}$ vectors by $\mathcal{H}(n)$ (note that the feasibility of an $\mathbf{h}$ vector depends on $n$ ). We see that $\forall \mathbf{h} \in \mathcal{H}(n), \cap_{l=1}^{L} E_{h_{l}}(n) \supseteq$ $\cap_{l=1}^{L} \cap_{j=1}^{J(n)} \cap_{i=1}^{h_{l}-1} A_{i, j}^{l}$, which implies that (analysing similar 


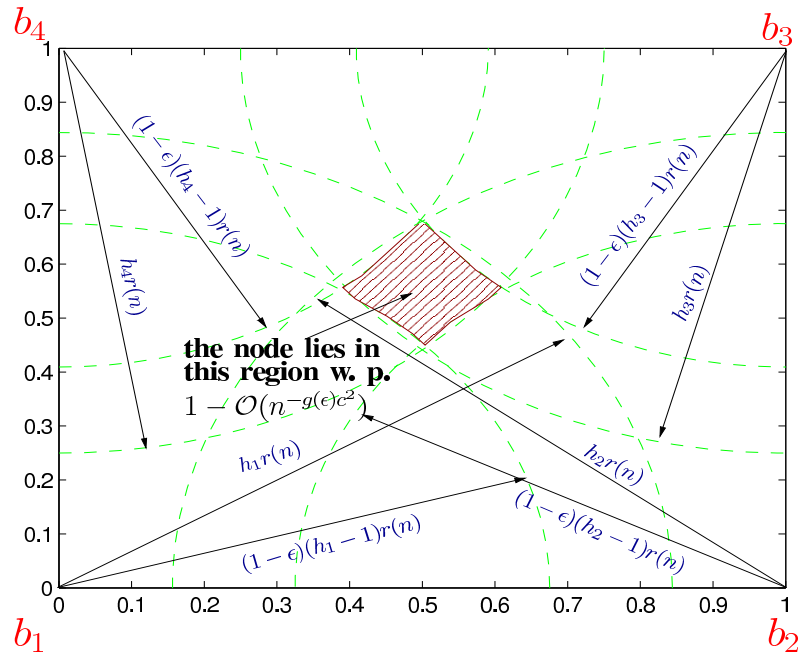

Figure 10: Graphical illustration of how Theorem 2 yields a location region for a node that is at a HD $h_{l}$ from anchor $b_{l}, 1 \leq l \leq 4$.

to Equation 2),

$$
\begin{aligned}
& \mathbb{P}^{n}\left(\cap_{l=1}^{L} E_{h_{l}}(n)\right) \\
& \geq \mathbb{P}^{n}\left(\cap_{l=1}^{L} \cap_{j=1}^{J(n)} \cap_{i=1}^{h_{l}-1} A_{i, j}^{l}\right) \\
& \geq 1-\sum_{l=1}^{L}\left(h_{l}-1\right)\left[\frac{\pi h_{l}}{2 \sqrt{1-p^{2}}}\right] n^{-q \sqrt{1-p^{2}} c^{2}} \\
& \Rightarrow \mathbb{P}^{n}\left(\cap_{l=1}^{L} E_{h_{l}}(n)\right)=1-\mathcal{O}\left(n^{-q \sqrt{1-p^{2}} c^{2}}\right)
\end{aligned}
$$

Hence we get the following theorem,

THEOREM 2. For a given $1>\epsilon>0$, and $r(n)=c \sqrt{\frac{\ln n}{n}}$, $c>\frac{1}{\sqrt{\pi}}, \forall \mathbf{h}=\left[h_{1}, \cdots, h_{l}, \cdots, h_{L}\right] \in \mathcal{H}(n)$,

$$
\mathbb{P}^{n}\left(\cap_{l=1}^{L} E_{h_{l}}(n)\right)=1-\mathcal{O}\left(\frac{1}{n^{g(\epsilon) c^{2}}}\right)
$$

where $g(\epsilon)=q(\epsilon) \sqrt{1-p^{2}(\epsilon)}$,

$$
p(\epsilon)=\frac{1-\epsilon+\sqrt{(1-\epsilon)^{2}+8}}{4}, q(\epsilon)=\frac{-3(1-\epsilon)+\sqrt{(1-\epsilon)^{2}+8}}{4}
$$

This theorem tells us that for a feasible $\mathbf{h}$, the node lies within the intersection of the annuli of inner and outer radii $(1-\epsilon)\left(h_{l}-1\right) r(n)$ and $h_{l} r(n)$ respectively, centred at anchors $b_{l}, 1 \leq l \leq L$, with a probability that scales as shown in the above theorem. A graphical illustration of this is shown in Figure 10 for $L=4$. This result motivates us to develop localisation schemes that use the hop-distance information from a few fixed anchor nodes. An example of the application of this theory to node localisation has been discussed in Chapter 6 of [5], where the author has shown a scheme of GPS-free localisation depending on the hop distances of the nodes from a finite number of fixed anchor nodes.

\section{HD-ED RELATIONSHIP IN RANDOM GE- OMETRIC GRAPHS: FIXED RADIUS}

The scaling of $r(n)$ with $n$ as shown in the previous section ensures asymptotic connectivity and increases the precision in localisation as $n \rightarrow \infty$. But in a wireless sensor network the radius $r$ of the RGG on which hop-distances are measured often corresponds to the radio range for a given transmit power, and hence does not decrease with $n$. So, it is meaningful to use a fixed radius $r$ for the RGG and it is denoted by $\mathcal{G}(\mathbf{v}, r)$. But for connectivity, we need to use number of nodes sufficient to make the network connected (i.e., the radius should scale with $n$ like $r(n)=c \sqrt{\frac{\ln n}{n}}, c>\frac{1}{\sqrt{\pi}}$, a constant; see [2] ), i.e., need at least $n_{0}=\inf \{n: r(n) \leq r\}$ nodes. Using a constant value for radius $r$, and redefining $E_{h_{l}}=\left\{\mathbf{v}:(1-\epsilon)\left(h_{l}-1\right) r \leq \underline{D}_{l}\left(\mathbf{v}, h_{l}\right) \leq \bar{D}_{l}\left(\mathbf{v}, h_{l}\right) \leq h_{l} r\right\}$, where the hop distance is measured on the RGG $\mathcal{G}(\mathbf{v}, r)$, we can show (along similar lines as for Equation 2),

$$
\begin{aligned}
1 & \geq \mathbb{P}^{n}\left(E_{h_{l}}\right) \\
& \geq \mathbb{P}^{n}\left(\cap_{j=1}^{J} \cap_{i=1}^{h_{l}-1} A_{i, j}^{l}\right) \\
& \geq 1-\left(h_{l}-1\right)\left[\frac{\pi h_{l}}{2 \sqrt{1-p^{2}}}\right] e^{-n q \sqrt{1-p^{2}} r^{2}}
\end{aligned}
$$

where $J \leq\left\lceil\frac{\pi h_{l}}{2 \sqrt{1-p^{2}}}\right\rceil$. Which implies, as $n \rightarrow \infty$,

$$
\begin{aligned}
1 & -\mathbb{P}^{n}\left(E_{h_{l}}\right) \\
& =\mathcal{O}\left(e^{-n q \sqrt{1-p^{2}} r^{2}}\right)
\end{aligned}
$$

Hence, $\lim _{n \rightarrow \infty} \mathbb{P}^{n}\left(E_{h_{l}}\right)=1$. So, for $L$ anchors, we will get $\forall \mathbf{h} \in \mathcal{H}$ (note that the set of feasible $\mathbf{h}$ vectors, $\mathcal{H}$, does not scale with $n$ in this case),

$$
\begin{aligned}
1 & -\mathbb{P}^{n}\left(\cap_{l=1}^{L} E_{h_{l}}\right) \\
& =\mathcal{O}\left(e^{-n q \sqrt{1-p^{2}} r^{2}}\right)
\end{aligned}
$$

Hence we get the following theorem.

Theorem 3. For a given $1>\epsilon>0$, and $r$ fixed, $\forall n \geq$ $n_{0}=\inf \{n: r(n) \leq r\}, \forall \mathbf{h}=\left[h_{1}, \cdots, h_{l}, \cdots, h_{L}\right] \in \mathcal{H}$,

$$
\mathbb{P}^{n}\left(\cap_{l=1}^{L} E_{h_{l}}\right)=1-\mathcal{O}\left(e^{-n g(\epsilon) r^{2}}\right)
$$

where $g(\epsilon)=q(\epsilon) \sqrt{1-p^{2}(\epsilon)}$,

$$
p(\epsilon)=\frac{1-\epsilon+\sqrt{(1-\epsilon)^{2}+8}}{4}, q(\epsilon)=\frac{-3(1-\epsilon)+\sqrt{(1-\epsilon)^{2}+8}}{4}
$$

Remark: We see that,

$$
\text { for all } \mathbf{h} \in \mathcal{H}, \lim _{n \rightarrow \infty} \mathbb{P}^{n}\left(\cap_{l=1}^{L} E_{h_{l}}\right)=1
$$

but with an exponential convergence rate compared to the power law scaling in the previous section. But it also says that the precision of localisation remains fixed at $r$ rather than increasing with $n$ like in the previous section.

\section{EXTENSION TO RANDOMISED LATTICE DEPLOYMENT}

In the previous sections we analysed the performance of ED-HD proportionality approximation for uniform i.i.d. deployment. In this section we will prove a similar result for 
the randomised lattice deployment. In randomised lattice node deployment, the unit area is split into $n$ cells each of area $\frac{1}{n}$, and in each cell exactly one node is deployed, uniformly over the cell area. The locations of the nodes in two different cells are independent of each other. We denote by $\mathbb{P}_{R L}^{(n)}($.$) the probability measure on \mathcal{A}^{n}$ so obtained (this is different from the uniform i.i.d. measure $\mathbb{P}^{n}($.$) ). We$ will show that, for this deployment also the above theorems hold. Here we consider the case in which the radius $r(n)$ of the RGG scales with $n$ as defined before. For fixed $r$, the theorem is valid too, which can be proved in a similar way as done in Section 5.

We have the following notation,

- $S_{k}^{i, j}$ : area belonging to the $i^{\text {th }}$ strip of $j^{\text {th }}$ blade (refer to Figures 6 and 7 ) of area $u(n) t(n)$ that falls in the $k^{t h}$ cell of the randomised lattice structure.

Thus, $\sum_{k=1}^{n} S_{k}^{i, j}=u(n) t(n), \forall 1 \leq i \leq h_{l}-1,1 \leq j \leq$ $J(n)$. Since a single node is uniformly distributed over each cell whose area is $\frac{1}{n}$,

$$
\mathbb{P}_{R L}^{n}\left(A_{i, j}^{l}{ }^{c}\right)=\prod_{k=1}^{n}\left(1-\frac{S_{k}^{i, j}}{\frac{1}{n}}\right)=\prod_{k=1}^{n}\left(1-n S_{k}^{i, j}\right)
$$

We see that,

$$
\sum_{k=1}^{n}\left(1-n S_{k}^{i, j}\right)=n(1-u(n) t(n)) \quad \begin{array}{ll}
\forall 1 \leq i \leq h_{l}-1 \\
& \forall 1 \leq j \leq J(n)
\end{array}
$$

Now, we know that the arithmetic mean is no smaller than the geometric mean. It follows that,

$$
\begin{aligned}
\mathbb{P}_{R L}^{n}\left(A_{i, j}^{l}{ }^{c}\right) & =\prod_{k=1}^{n}\left(1-n S_{k}^{i, j}\right) \leq\left(\frac{1}{n} \sum_{k=1}^{n}\left(1-n S_{k}^{i, j}\right)\right)^{n} \\
& =(1-u(n) t(n))^{n}
\end{aligned}
$$

Hence we get (analysing similar to Equation 2, 3, 4 and 5) the following theorem,

THEOREM 4. For a given $1>\epsilon>0$, and $r(n)=c \sqrt{\frac{\ln n}{n}}$, $c>\frac{1}{\sqrt{\pi}}, \mathbb{P}_{R L}^{n}\left(E_{h_{l}}(n)\right)=1-\mathcal{O}\left(\frac{1}{n^{g(\epsilon) c^{2}}}\right)$,

where $g(\epsilon)=q(\epsilon) \sqrt{1-p^{2}(\epsilon)}$,

$p(\epsilon)=\frac{1-\epsilon+\sqrt{(1-\epsilon)^{2}+8}}{4}, q(\epsilon)=\frac{-3(1-\epsilon)+\sqrt{(1-\epsilon)^{2}+8}}{4}$.

Hence, $\lim _{n \rightarrow \infty} \mathbb{P}_{R L}^{n}\left(E_{h_{l}}(n)\right)=1$. Following a similar analysis as in Section 4.2 for $L$ anchors, we can state the following theorem for randomised lattice node deployment.

TheOREM 5. For a given $1>\epsilon>0$, and $r(n)=c \sqrt{\frac{\ln n}{n}}$, $c>\frac{1}{\sqrt{\pi}}, \forall \mathbf{h}=\left[h_{1}, \cdots, h_{l}, \cdots, h_{L}\right] \in \mathcal{H}(n)$,

$$
\mathbb{P}_{R L}^{n}\left(\cap_{l=1}^{L} E_{h_{l}}(n)\right)=1-\mathcal{O}\left(\frac{1}{n^{g(\epsilon) c^{2}}}\right)
$$

where $g(\epsilon)=q(\epsilon) \sqrt{1-p^{2}(\epsilon)}$,

$$
p(\epsilon)=\frac{1-\epsilon+\sqrt{(1-\epsilon)^{2}+8}}{4}, q(\epsilon)=\frac{-3(1-\epsilon)+\sqrt{(1-\epsilon)^{2}+8}}{4}
$$

\section{ED BOUND ON A SINGLE BLADE WITH POISSON DEPLOYMENT}

Here we consider another kind of deployment, where we pick the number of nodes with the distribution Poisson $(n)$ and deploy these nodes uniformly over the area $\mathcal{A}$. The number of nodes falling in $\mathcal{A}$ is a random variable with mean $n$, and since we are throwing the picked nodes uniformly over $\mathcal{A}$, the nodes falling in disjoint areas are independent and Poisson distributed with rate proportional to the area considered. Hence, for disjoint strips with area $u(n) t(n)$ each and the number of node selection being Poisson $(n)$, the number of nodes falling in each strip is Poisson $(n u(n) t(n))$, independent and identically distributed. Let the probability law associated with this kind of deployment be denoted by $\mathbb{P}_{\text {Poo }}^{n}($.$) . Let us focus our attention to a certain blade \mathcal{B}_{j}^{l}$ as shown in Figure 7 pivoted at the anchor location $b_{l}$. We also denote the maximum and minimum Euclidean distance travelled by a $h_{l}$ hop path within this blade by $\bar{D}_{l}^{\mathcal{B}_{j}^{l}}\left(\mathbf{v}, h_{l}\right)$ and $\underline{D}_{l}^{\mathcal{B}_{j}^{l}}\left(\mathbf{v}, h_{l}\right)$ respectively. Now, ensuring at least one node in each of the $h_{l}-1$ strips of $\mathcal{B}_{j}^{l}$ will ensure the event $E_{h_{l}}^{\mathcal{B}_{j}^{l}}(n)=$ $\left\{\mathbf{v}:(1-\epsilon)\left(h_{l}-1\right) r(n) \leq \underline{D}_{l}^{\mathcal{B}_{j}^{l}}\left(\mathbf{v}, h_{l}\right) \leq \bar{D}_{l}^{\mathcal{B}_{j}^{l}}\left(\mathbf{v}, h_{l}\right) \leq h_{l} r(n)\right\}$ also occurs. So, we have for the given $\epsilon>0$,

$$
\begin{aligned}
1 & \geq \mathbb{P}_{P o}^{n}\left(E_{h_{l}}^{\mathcal{B}_{j}^{l}}(n)\right) \\
& \geq \mathbb{P}_{P o}^{n}\left(\cap_{i=1}^{h_{l}-1} A_{i, j}^{l}\right) \\
& =\left(1-e^{-n u(n) t(n)}\right)^{h_{l}-1} \\
& =\left(1-n^{-c^{2} q \sqrt{1-p^{2}}}\right)^{h_{l}-1}
\end{aligned}
$$

The second inequality comes because $\left\{\mathbf{v} \in \cap_{i=1}^{h_{l}-1} A_{i, j}^{l}\right\} \subseteq$ $\left\{\mathbf{v} \in E_{h_{l}}^{\mathcal{B}_{j}^{l}}(n)\right\}$ and the first equality comes because of the independence of the number of nodes due to Poisson deployment and disjoint strips. Since in this deployment we are not using the union bound, the expression for probability is exact. Hence the bound on the probability of the event $E_{h_{l}}^{\mathcal{B}_{j}^{l}}(n)$ is tighter, yet the rate of convergence follows the power law $\left(e^{-n u(n) t(n)}=n^{-q \sqrt{1-p^{2}} c^{2}}\right)$.

\section{SIMULATION RESULTS}

In this section, we illustrate Theorem 1 through some simulation examples. We deploy $n$ nodes in the uniform i.i.d. fashion on the unit square $\mathcal{A}$, and form the geometric graph $\mathcal{G}(\mathbf{v}, r(n))$, where $r(n)=\frac{4}{\sqrt{\pi}} \sqrt{\frac{\ln n}{n}}$. We also have 4 anchors at the 4 corners of $\mathcal{A}$.

\section{Illustration of Theorem 1 with increasing $n$ for a} fixed $\epsilon$ and HD:

We fix $\epsilon=0.4$ and hop-distance $h_{1}=5$ from anchor $b_{1}$ located at the bottom-left corner of the unit square $\mathcal{A}$. The results are summarised in Table 1 and illustrate how the theoretical bounds given in Theorem 1 become tighter as we increase the number of nodes $n$, keeping the hop-distance $h_{1}$ and $\epsilon$ fixed.

Figures 11 and 12 show the theoretical bounds given by Theorem 1, and only those nodes are shown that have a hopdistance $h_{1}=5$ from anchor $b_{1}$, for 1000 and 5000 nodes, 


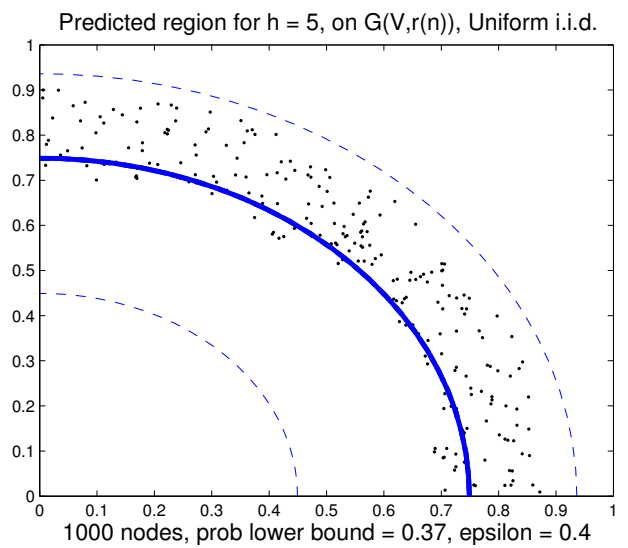

Figure 11: Locations of nodes that are 5 hops away from an anchor $\left(b_{1}\right)$ at the origin; 1000 nodes were deployed in a uniform i.i.d. fashion. The thin dashed lines show the ED bounds given by Theorem 1, the thick solid line shows ED $\left(h_{1}-\right.$ 1) $r(n)$ from $b_{1} ; \epsilon=0.4, \mathbb{P}^{n}\left(E_{1}(n)\right) \geq 0.37$. $r(n)=$ $\frac{4}{\sqrt{\pi}} \sqrt{\frac{\ln n}{n}}$.

Table 1: Euclidean Distance Lower Bound (EDLB) $=(1-\epsilon)\left(h_{1}-1\right) r(n)$ and Euclidean Distance Upper Bound (EDUB) $=h_{1} r(n)$ are found from Theorem 1. $\underline{D}_{1}$ and $\bar{D}_{1}$ are the maximum and minimum EDs from anchor 1 given the hop-distance $h_{1}=5$. The theoretical Probability Lower Bound (PLB) $=$ $1-\left(h_{1}-1\right)\left[\frac{\pi h_{1}}{2 \sqrt{1-p^{2}(\epsilon)}}\right] e^{-n g(\epsilon) r^{2}(n)}$, and the Empirical Probability (EP) is found from this experiment. $r(n)=\frac{4}{\sqrt{\pi}} \sqrt{\frac{\ln n}{n}}, \epsilon=0.4$.

\begin{tabular}{||c|c|c|c|c|c|c|c||}
\hline$n$ & $r(n)$ & EDLB & $\underline{D}_{1}$ & $\bar{D}_{1}$ & EDUB & PLB & EP \\
\hline \hline 1000 & 0.1876 & 0.4494 & 0.6934 & 0.9053 & 0.9362 & 0.37 & 1 \\
\hline 2000 & 0.1391 & 0.3336 & 0.5196 & 0.6678 & 0.6950 & 0.61 & 1 \\
\hline 3000 & 0.1166 & 0.2796 & 0.4313 & 0.5590 & 0.5826 & 0.70 & 1 \\
\hline 4000 & 0.1028 & 0.2465 & 0.3761 & 0.4929 & 0.5136 & 0.75 & 1 \\
\hline 5000 & 0.0931 & 0.2235 & 0.3428 & 0.4559 & 0.4655 & 0.79 & 1 \\
\hline 6000 & 0.0859 & 0.2062 & 0.3123 & 0.4191 & 0.4295 & 0.81 & 1 \\
\hline \hline
\end{tabular}

respectively. We notice that, for this range of values of $n$, while the maximum Euclidean distance, $\bar{D}_{1}$, is quite close to the upper bound (obtained from the triangle inequality), the lower bound is loose when compared to the minimum Euclidean distance, $\underline{D}_{1}$. Indeed, all the node locations lie well within the bounds, and, in fact, $(h-1) r(n)$ (the thick solid quarter circle in the figures) could serve as a good approximation to $\underline{D}_{1}$, but this bound is certainly not met with a high probability. The theoretical lower bound on the probability of the upper and lower bounds being respected is seen to be increasing to 1 as the number of nodes, $n$, is increased.

Illustration of Theorem 1 with decreasing HD for a fixed $n$ and a fixed lower bound on probability:

We have fixed the number of nodes $n=5000$ and also fixed

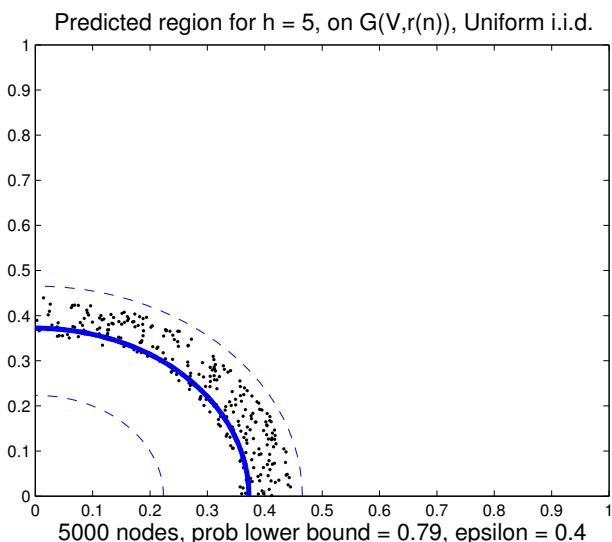

Figure 12: Locations of nodes that are 5 hops away from an anchor $\left(b_{1}\right)$ at the origin; 5000 nodes were deployed in a uniform i.i.d. fashion. The thin dashed lines show the ED bounds given by Theorem 1, the thick solid line shows ED $\left(h_{1}-\right.$ 1) $r(n)$ from $b_{1} ; \epsilon=0.4, \mathbb{P}^{n}\left(E_{1}(n)\right) \geq 0.79$. $r(n)=$ $\frac{4}{\sqrt{\pi}} \sqrt{\frac{\ln n}{n}}$.

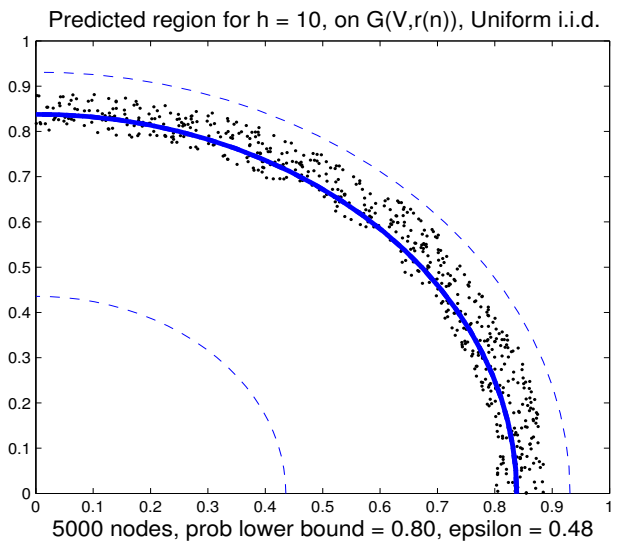

Figure 13: Locations of nodes that are 10 hops away from an anchor $\left(b_{1}\right)$ at the origin; 5000 nodes were deployed in a uniform i.i.d. fashion. The thin dashed lines show the ED bounds given by Theorem 1, the thick solid line shows ED $\left(h_{1}-1\right) r(n)$ from $b_{1}$; $\mathbb{P}^{n}\left(E_{1}(n)\right) \geq 0.80 . r(n)=\frac{4}{\sqrt{\pi}} \sqrt{\frac{\ln n}{n}}$.

the lower bound on probability that the node lies within the bound of $\left[(1-\epsilon)\left(h_{1}-1\right) r(n), h_{1} r(n)\right]$ (as given by Theorem 1 ) at 0.80 . Figures 13, 14 and 15 show that as we decrease the hop-distance $h_{1}$, the bound on the ED becomes tighter, which implies that if we keep the lower bound fixed, the $\epsilon$ that achieves that lower bound will be smaller for smaller hop-distances, as predicted by Theorem 1 .

Illustration of convergence in probability for the ge- 


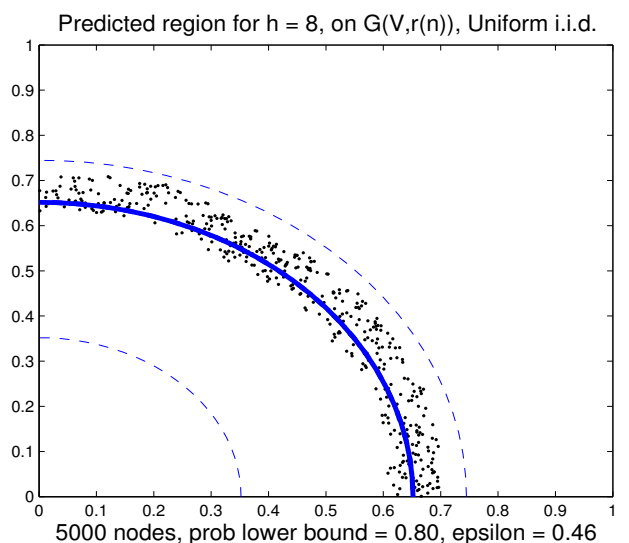

Figure 14: Locations of nodes that are 8 hops away from an anchor $\left(b_{1}\right)$ at the origin; 5000 nodes were deployed in a uniform i.i.d. fashion. The thin dashed lines show the ED bounds given by Theorem 1, the thick solid line shows ED $\left(h_{1}-1\right) r(n)$ from $b_{1}$; $\mathbb{P}^{n}\left(E_{1}(n)\right) \geq 0.80 . r(n)=\frac{4}{\sqrt{\pi}} \sqrt{\frac{\ln n}{n}}$.

Table 2: Radius $r=0.1, h_{1}=5, \epsilon=0.36$. The theoretical PLB $=1-\left(h_{1}-1\right)\left[\frac{\pi h_{1}}{2 \sqrt{1-p^{2}(\epsilon)}}\right] e^{-n g(\epsilon) r^{2}}$. Abbreviations are as defined in Table 1 .

\begin{tabular}{||c|c|c|c|c|c|c|}
\hline$n$ & EDLB & $\underline{D}_{1}$ & $\bar{D}_{1}$ & EDUB & PLB & EP \\
\hline \hline 1000 & 0.2560 & 0.3483 & 0.4574 & 0.5000 & 0.0000 & 1 \\
\hline 2000 & 0.2560 & 0.3479 & 0.4677 & 0.5000 & 0.0000 & 1 \\
\hline 3000 & 0.2560 & 0.3775 & 0.4835 & 0.5000 & 0.0000 & 1 \\
\hline 4000 & 0.2560 & 0.3741 & 0.4843 & 0.5000 & 0.2906 & 1 \\
\hline 5000 & 0.2560 & 0.3831 & 0.4897 & 0.5000 & 0.7733 & 1 \\
\hline 6000 & 0.2560 & 0.3705 & 0.4826 & 0.5000 & 0.9275 & 1 \\
\hline \hline
\end{tabular}

ometric graph with fixed radius:

We fix the radius of the graph $\mathcal{G}(\mathbf{v}, r), r=0.1$ and take $h_{1}=5, \epsilon=0.36$. The simulation results are summarised in Table 2, which shows that for smaller $n$, the lower bound of probability (as given by Equation 6) is weak, but the convergence rate, due to its exponential nature, is very rapid with increase in $n$.

\section{CONCLUSION}

In this paper, we have formally studied the often used heuristic that HD on a geometric graph on a plane is proportional to the ED. For arbitrary 2-D node placements, we saw in Lemma 2, that for HD $h \geq 2, r<\mathrm{ED} \leq h r$ and this is not even roughly proportional to HD. For homogeneous random deployments, we have found that for given HD $h$, $(1-\epsilon)(h-1) r<\mathrm{ED} \leq h r$ w.h.p. (this is true even for a nonhomogeneous node placement with a positive density over all points of the area). Our proof techniques rely on a certain geometric construction and the union bound. The parameter $\epsilon$ provides a trade-off between ED-HD proportionality and the rate of convergence of the desired probability to 1 . This result holds for both uniform i.i.d. (Theorem 1) and randomised lattice (Theorem 4) node placements. We also

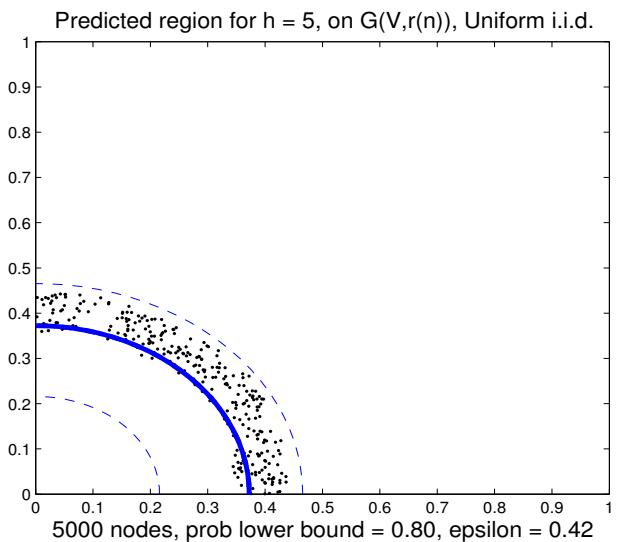

Figure 15: Locations of nodes that are 5 hops away from an anchor $\left(b_{1}\right)$ at the origin; 5000 nodes were deployed in a uniform i.i.d. fashion. The thin dashed lines show the ED bounds given by Theorem 1, the thick solid line shows ED $\left(h_{1}-1\right) r(n)$ from $b_{1}$; $\mathbb{P}^{n}\left(E_{1}(n)\right) \geq 0.80 . r(n)=\frac{4}{\sqrt{\pi}} \sqrt{\frac{\ln n}{n}}$.

provide Theorems 2, 3 and 5, that can be useful for GPSfree localisation. Simulation results show that the actual probability of ED-bounds being respected is much closer to 1 than that provided by our bounding arguments. Given an $\mathrm{HD}, h$, the simulations suggest that $[(h-1) r, h r]$ could serve as a good approximation to the interval in which the EDs of the nodes lie; however, this might not be a high probability bound. In our future work, we seek more precise bounds on the ED with a provably higher probability of being respected.

\section{Acknowledgements}

This work was supported by a research grant from DRDO, Government of India. We are grateful for useful discussions with Rajesh Sundaresan, Srikant Iyer, Srivathsa Acharya, N. E. Venkatesan and P. Vijay Kumar.

\section{REFERENCES}

[1] Stefan Dulman, Michele Rossi, Paul Havinga, and Michele Zorzi. On the hop count statistics for randomly deployed wireless sensor networks. Int. J. Sensor Networks, I(1/2), 2006.

[2] Piyush Gupta and P. R. Kumar. Critical power for asymptotic connectivity in wireless networks. Stochastic Analysis, Control, Optimization and Applications, 1998.

[3] Mo Li and Yunhao Liu. Rendered Path: Range-free localization in anisotropic sensor networks with holes. In Proceedings of Mobicom. ACM, September 2007.

[4] Radhika Nagpal, Howard Shrobe, and Jonathan Bachrach. Organizing a global coordinate system from local information on an ad hoc sensor network. In IPSN. IEEE, 2003

[5] Swaprava Nath. Self Organisation in Random Geometric Graph models of Wireless Sensor Networks. Master's thesis, Indian Institute of Science, Bangalore, INDIA, June 2008. 


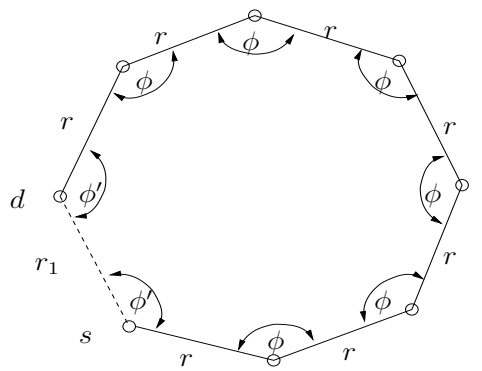

Figure 16: $\phi=\frac{\left(h_{l}-1\right) \pi}{h_{l}+1}+\delta$ and $\phi^{\prime}=\frac{\left(h_{l}-1\right) \pi}{h_{l}+1}-\frac{\left(h_{l}-1\right)}{2} \delta$.

[6] D. Niculescu and B. Nath. Ad hoc positioning system (aps). In IEEE Globecom. IEEE, Nov. 2001.

[7] M. D. Penrose. Random Geometric Graphs. Oxford University Press, 2003.

[8] Serdar Vural and Eylem Ekici. Analysis of hop-distance relationship in spatially random sensor networks. In Mobihoc. ACM, 2005.

[9] Sungwon Yang, Jiyoung Yi, and Hojung Cha. HCRL: A Hop-Count-Ratio based Localization in wireless sensor networks. In IEEE SECON. IEEE, 2007.

\section{APPENDIX}

Proof of Lemma 1: The setting for this lemma is as described in Section 3 and as depicted in Figure 5. We had an $h_{l}+1$ sided regular polygon with sides of length $r$. We deleted a certain edge $s d$ and increased all angles except the two adjacent to the deleted edge by a small amount $\delta$. The resulting figure is as shown in Figure 16. The length of edge $s d$ in this new figure is $r_{1}$. We restate the lemma.

Lemma: For $h_{l}>2$ and $0<\delta<\frac{4 \pi}{h_{l}+1}, r_{1}>r$

Proof: Each of the internal angles except the adjacent angles of $s d$ is $\phi=\frac{\left(h_{l}-1\right) \pi}{h_{l}+1}+\delta$ (see Figure 16) and angles adjacent to $s d$ are $\phi^{\prime}=\frac{1}{2}\left[\right.$ total internal angle $\left.-\left(h_{l}-1\right) \times \phi\right]=$ $\frac{1}{2}\left[\left(h_{l}-1\right) \pi-\left(h_{l}-1\right)\left(\frac{\left(h_{l}-1\right) \pi}{h_{l}+1}+\delta\right)\right]=\frac{\left(h_{l}-1\right) \pi}{h_{l}+1}-\frac{\left(h_{l}-1\right)}{2} \delta$, $h_{l}>2$. It can be easily proved ${ }^{4}$ that the angle bisectors of all these internal angles meet at the point $c$ as shown in Figure 17. Now, we apply sine rule in the triangles $\triangle s d c$ and $\triangle s p_{1} c$, where $p_{1}$ is the adjacent node of $s$ other than $d$ (see Figure 17). $z$ is the length of the line segment connecting $s$ and $c$. For $\triangle s d c$,

$$
\begin{aligned}
\frac{r_{1}}{\sin \left(\pi-\phi^{\prime}\right)} & =\frac{z}{\sin \frac{\phi^{\prime}}{2}} \\
z & =\frac{r_{1}}{2 \cos \frac{\phi^{\prime}}{2}}
\end{aligned}
$$

and for $\triangle s p_{1} c$

$$
\begin{aligned}
\frac{z}{\sin \frac{\phi}{2}} & =\frac{r}{\sin \left(\pi-\frac{\phi}{2}-\frac{\phi^{\prime}}{2}\right)} \\
z & =\frac{r \sin \frac{\phi}{2}}{\sin \left(\frac{\phi+\phi^{\prime}}{2}\right)}
\end{aligned}
$$

${ }^{4}$ It can be shown that $\left(\pi-\phi^{\prime}\right)+2\left(\pi-\frac{\phi}{2}-\frac{\phi^{\prime}}{2}\right)+\left(h_{l}-2\right)(\pi-$ $\phi)=2 \pi$

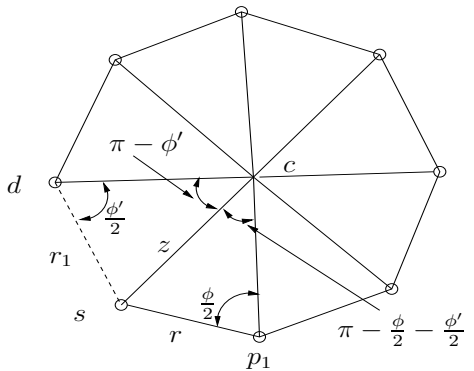

Figure 17: $c$ is the point where the angle bisectors meet.

Eliminating $z$ from these two equations, we get,

$$
r_{1}=\frac{2 r \sin \frac{\phi}{2} \cos \frac{\phi^{\prime}}{2}}{\sin \frac{\phi}{2} \cos \frac{\phi^{\prime}}{2}+\cos \frac{\phi}{2} \sin \frac{\phi^{\prime}}{2}}
$$

Now we have, $\frac{\phi-\phi^{\prime}}{2}=\frac{h_{l}+1}{4} \delta$ and $\frac{\phi+\phi^{\prime}}{2}=\frac{\left(h_{l}-1\right) \pi}{h_{l}+1}-\frac{\left(h_{l}-3\right)}{4} \delta$. Hence, to show,

$$
\begin{aligned}
& r_{1}>r \\
\Rightarrow & r_{1}-r>0 \\
\Rightarrow & r \frac{\sin \frac{\phi-\phi^{\prime}}{2}}{\sin \frac{\phi+\phi^{\prime}}{2}}>0 \\
\Rightarrow & r \frac{\sin \frac{h_{l}+1}{4} \delta}{\sin \left(\frac{\left(h_{l}-1\right) \pi}{h_{l}+1}-\frac{\left(h_{l}-3\right)}{4} \delta\right)}>0
\end{aligned}
$$

For the numerator to be positive, $\pi>\frac{h_{l}+1}{4} \delta>0 \Rightarrow \frac{4 \pi}{h_{l}+1}>$ $\delta>0$. For the denominator, the condition is $\frac{4\left(h_{l}-1\right) \pi}{\left(h_{l}+1\right)\left(h_{l}-3\right)}>$ $\delta>0$, for $h_{l} \geq 3$. Together, the condition on $\delta$ is $\frac{4 \pi}{h_{l}+1}>$ $\delta>0$, to have $r_{1}>r$, for $h_{l}>2$ (since $\frac{h_{l}-1}{h_{l}-3}>1$ and so, $\left.\frac{4\left(h_{l}-1\right) \pi}{\left(h_{l}+1\right)\left(h_{l}-3\right)}>\frac{4 \pi}{h_{l}+1}, \forall h_{l} \geq 3\right)$. Hence proved. 